\title{
Space Weather Activities of IONOLAB Group Using TNPGN GPS Network
}

\author{
Bahadır AKTUĞ \\ Onur LENK \\ Mustafa KURT \\ Erdem PARMAKSIZ \\ Soner OZDEMIR \\ Department of Geodesy \\ General Command of Mapping \\ 06100 Cebeci, Ankara, Turkey \\ bahadir.aktug@hgk.msb.gov.tr
}

\author{
Feza ARIKAN \\ Umut SEZEN \\ Cenk TOKER \\ Department of Electrical and Electronics Engineering \\ Hacettepe University \\ 06800, Beytepe, Ankara, Turkey \\ arikan@hacettepe.edu.tr \\ Orhan ARIKAN \\ Department of Electrical and Electronics Engineering \\ Bilkent University \\ 06800, Bilkent, Ankara, Turkey \\ oarikan@ee.bilkent.edu.tr
}

\begin{abstract}
Characterization and constant monitoring of variability of the ionosphere is of utmost importance for the performance improvement of $\mathrm{HF}$ communication, Satellite communication, navigation and guidance systems, Low Earth Orbit (LEO) satellite systems, Space Craft exit and entry into the atmosphere and space weather. Turkish National Permanent GPS Network (TNPGN) is the Reference Station Network of 146 continuously-operating GNSS stations of which are distributed uniformly across Turkey and North Cyprus Turkish Republic since May 2009. IONOLAB group is currently investigating new techniques for space-time interpolation, and automatic mapping of TEC through a TUBITAK research grant. It is utmost importance to develop regional stochastic models for correction of ionospheric delay in geodetic systems and also form a scientific basis for communication link characterization. This study is a brief summary of the efforts of IONOLAB group in monitoring of space weather, and correction of geodetic positioning errors due to ionosphere using TNPGN.
\end{abstract}

Keywords-GPS, Ionosphere, Space Weather, Monitoring of Ionospheric Variability, TEC, TEC Mapping

\section{INTRODUCTION}

Space Weather (SW) is the concept of changing environmental conditions in outer space and its effects on Earth and technological systems. SW is a consequence of the solar activities and the coupling of solar energy on Earth's atmosphere due to the Earth's magnetic field. The monitoring and prediction of SW has utmost importance for $\mathrm{HF}$ communication, Satellite communication, navigation and guidance systems, Low Earth Orbit (LEO) satellite systems, Space Craft exit and entry into the atmosphere. Ionosphere is the plasma layer of the atmosphere that is ionized by solar radiation and it is a key player of SW. Ionosphere is a temporally and spatially varying, dispersive, anisotropic and inhomogeneous medium that is characterized primarily by its electron density distribution. IONOLAB is a group of researchers of various disciplines, getting together to handle challenges of the Earth's ionosphere. The team has researchers from Hacettepe University and Bilkent University, Department of Electrical and Electronics Engineering and General Command of Mapping of Turkish Army. Turkish National Permanent GPS Network (TNPGN) is the Reference Station Network of 146 continuously-operating GNSS stations of which are distributed uniformly across Turkey and North Cyprus Turkish Republic since May 2009 (Figure 1). TNPGN provides a wide variety of possibilities for active research not only in geodetics but also in areas from geology to tropospheric and ionospheric characterization.

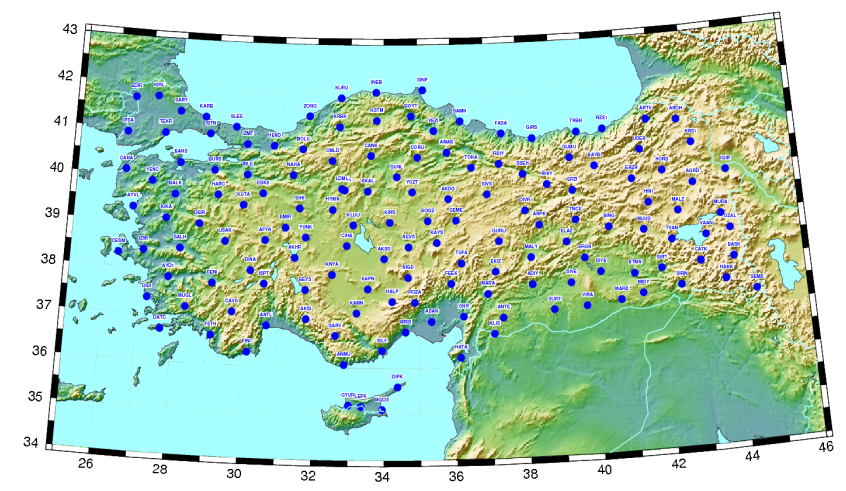

Figure 1: 146 GPS stations in TNPGN Network. 
Ionosphere is the main source of error for navigation and positioning systems and it can also cause major disturbance for satellite communication. Therefore, characterization and constant monitoring of variability of the ionosphere is of utmost importance for the performance improvement of these aforementioned systems and space weather. IONOLAB group has been active in estimation of Total Electron Content (TEC) using single station GPS recordings in IGS network, regional spatial interpolation (mapping) of TEC, global Computerized Ionospheric Tomography (CIT), statistical analysis of TEC, and investigation of lithosphere-ionosphere coupling through seismic activity. The techniques that have been developed for various purposes can be applied to any GPS station in IGS or EUREF network. With the new TNPGN, it is observed that there are various prospective areas in processing GPS recordings for higher accuracy and reliability in characterization of the regional ionosphere. The first one of these areas is an efficient and effective signal processing algorithm for computation of TEC using 146 GPS stations operating in a network. This goal will be achieved by modification of the physical International Reference Ionosphere model extending to Plasmasphere (IRI-Plas) with the IONOLAB-TEC estimates. A new parametric tomographic reconstruction technique working on the modified IRI-Plas will provide an updated regional ionosphere model. The second task is the monitoring of the TEC using optimized and automatic spatial interpolation, i.e. mapping. IONOLAB group is currently investigating a novel method in introducing anisotropicity into Ordinary Kriging for TEC mapping and an accurate temporal model for the semivariogram function for TNPGN Network. In the following section, a selection of the new results and investigations by IONOLAB group on TEC computation, TEC mapping and statistical analysis of TEC are presented.

\section{REGIONAL SPACE-TIME PARAMETRIC MODELING OF IONOSPHERE}

One of the main observable parameters of ionosphere is Total Electron Content (TEC) and it is defined as the number of free electrons in a column of $1 \mathrm{~m}^{2}$ base joining the ground GPS reference station and the GPS satellite. The unit of TEC is TECU corresponding to $10^{16}$ electrons $/ \mathrm{m}^{2}$. IONOLAB group has developed an important tool in estimation of TEC with a single GPS station, IONOLAB-TEC [1-4]. IONOLAB-TEC provides reliable and robust estimates for all latitudes and both calm and disturbed days by using RINEX, IONEX and satellite ephemeris data provided from the IGS centers. IONOLABTEC consists of a regularized signal estimation algorithm which combines signals from all GPS satellites for a given instant and a given receiver, for a desired time period or for 24 hours, with $30 \mathrm{~s}$ time resolution. IONOLAB-TEC values also include the receiver differential code bias (DCB) for each GPS station estimated uniquely by the IONOLAB-BIAS algorithm. The web based computation program is written in JAVA and it is provided both in Turkish and English at www.ionolab.org. An example of IONOLAB-TEC for three IGS stations ista (Istanbul), tubi (Tubitak, Gebze, Kocaeli), and ankr (Ankara) is presented in Figure 2 for August 30, 2009. That is the most positively disturbed day in August where Kp and Dst indices for that day indicate that there is a sudden commencement of a geomagnetic storm.

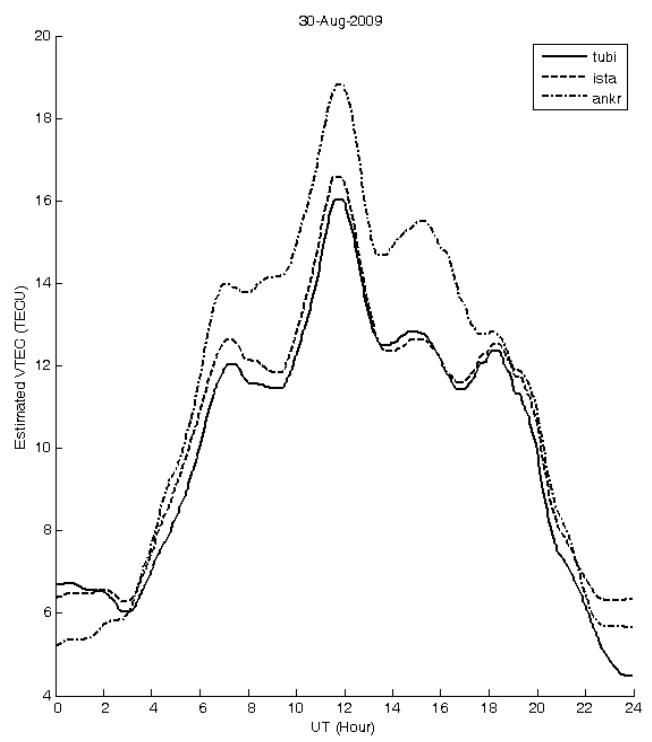

Figure 2: IONOLAB-TEC for August 30, 2009 for ankr (dashdot), ista (dashed) and tubi (solid) IGS stations.

The experimental hourly probability density functions (pdf) for some GPS reference station sites are investigated according to the method presented in [5] between 2001 and 2008, and it is been observed that yearly pdf distributions are Weibull distributed for hours that correspond to sunrise and sunset. Other hours are Lognormally distributed. As for years with low solar activity, all hours tend to be Lognormally distributed. In Figure 3, the Lognormal pdf first and second parameters are presented for years 2001 to 2008 with respect to Local Time (LT, UT+2h) for ankr station.
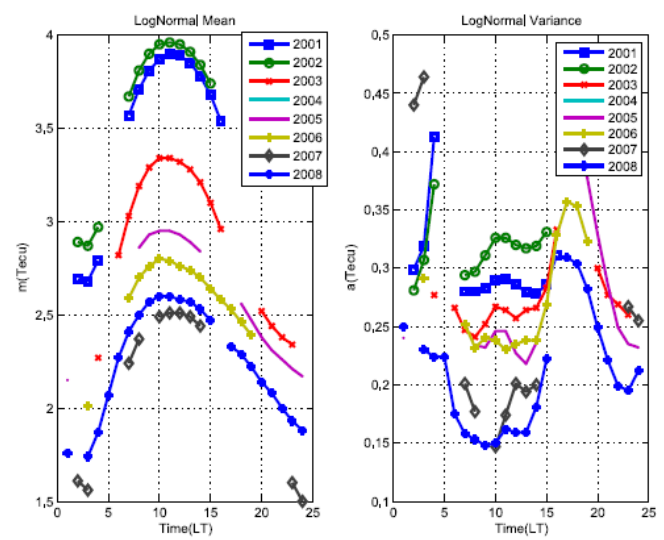

Figure 3: Lognormal pdf first and second parameters for ankr.

The hourly pdf values are obtained in a similar manner for 146 TNPGN stations for years 2009 and 2010. These pdf distributions will be used in space time stochastic modeling of ionosphere over Turkey. 
The east-west extend of Turkey indicates a major anisotropy in TEC distribution during sunrise and sunset hours. A north-south anisotropy is also observed for hours with full sun exposure and during night hours. IONOLAB group is currently investigating a novel method in introducing anisotropicity into Ordinary Kriging for TEC mapping and an accurate temporal model for the semivariogram function for TNPGN Network. The IONOLAB-TEC from 146 stations can be spatially interpolated using Ordinary Kriging and Universal Kriging. Optimum theoretical semivariogram functions can be obtained by investigating synthetic TEC maps generated for this purpose. In Figure 4, an example synthetic surface having a linear trend with additive small scale variability and TNPGN sampling is presented.

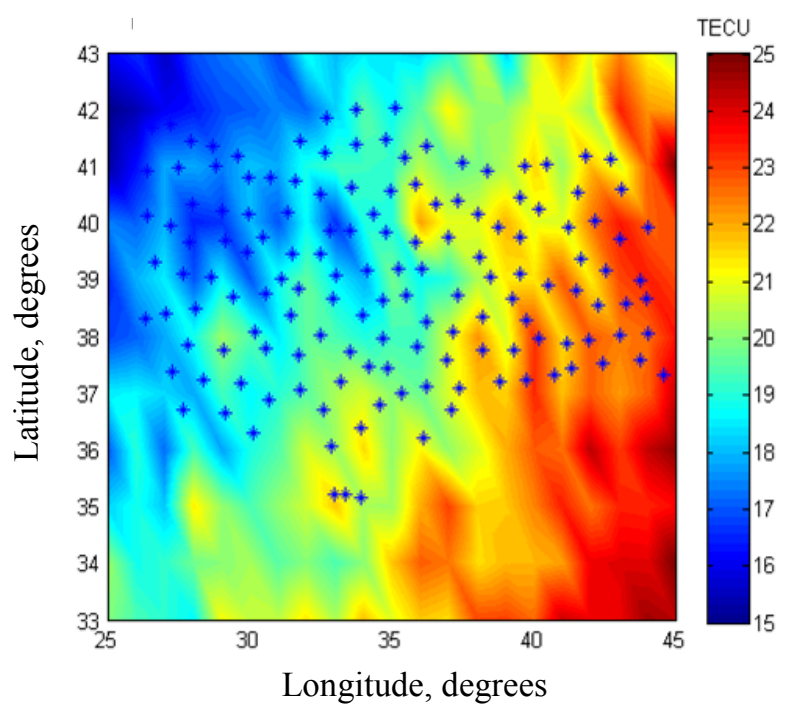

Figure 4: Synthetic TEC surface, linear trend with small scale variabilities using TNPGN sampling.

Experimental semivariogram functions for Ordinary Kriging and Universal Kriging with Linear Trend for the surface presented above are provided in Figure $5 \mathrm{a}$, and $5 \mathrm{~b}$ respectively.

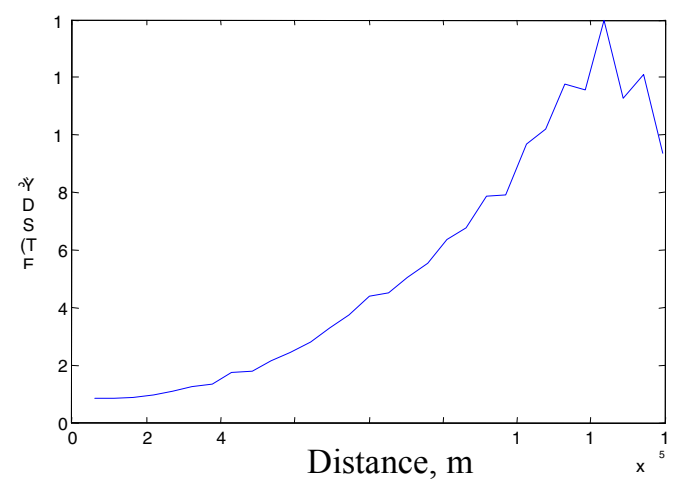

a)

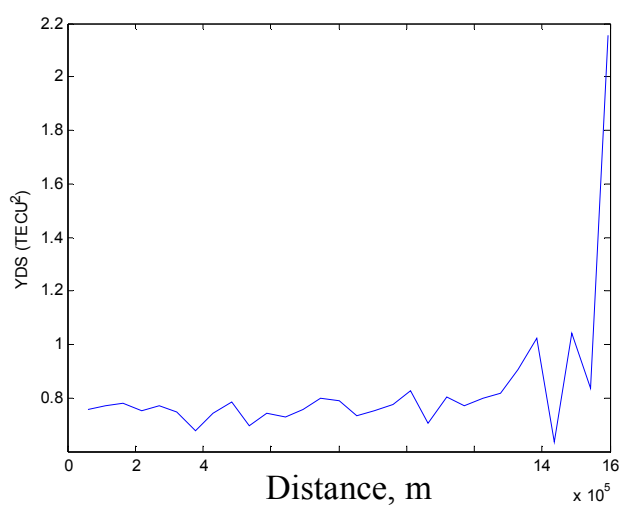

b)

Figure 5: Experimental semivariogram functions for a) Ordinary Kriging b) Universal Kriging with Linear Trend

In Figure 6, TEC map of Turkey, using TNPGN stations for July 22, 2009 at 1200 UT, obtained using isotropic Ordinary Kriging, is presented. A linear north to south TEC variation is very apparent from this map. Black dots indicate location of TNPGN stations.

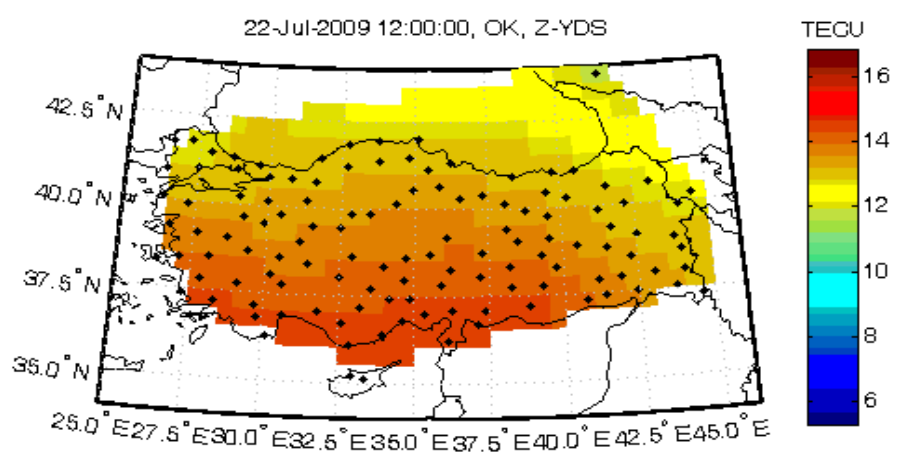

Figure 6: TEC map of Turkey using TNPGN on July 22, 2009, at 1200 UT with Ordinary Kriging.

TEC maps will be generated automatically according to a specialized, optimum temporal update period (TUP). This way, the ionospheric variability will be monitored and characterized accurately with low computational complexity and data storage. A random space-time model of ionosphere over Turkey can be developed using the TEC maps with optimum TUP. Active seismicity will also be monitored using the TNPGN network by observing and monitoring the disturbances in the regional ionosphere due to tectonic movements. The IONOLAB-TEC values are also used in ionospheric modeling programs such as IRI-Plas [6] for investigating the possibilities of parametric tomographic reconstruction of ionospheric electron density over Turkey.

\section{CONCLUSION}

Monitoring of ionosphere with a dense, homogeneous GPS network provides important possibilities in characterization and modeling of electron density and TEC. In this study, activities of IONOLAB group are introduced for Parametric CIT, TEC 
Mapping and development of a random space-time model using the new GPS network TNPGN. An updated statistical space-time model of regional ionosphere will enable classification of disturbances and prediction of parameters of SW. Also, statistical channel models for satellite links and HF communication can be obtained in the future.

\section{ACKNOWLEDGMENT}

This study is supported by TUBITAK EEEAG Grant No: 109E055.

\section{REFERENCES}

[1] Arikan, F., Erol, C.B. and Arikan, O., "Regularized estimation of vertical total electron content from Global Positioning System data", Journal of Geophysical Research-Space Physics, vol. 108(A12), 1469$1480,2003$.

[2] Arikan, F., Erol, C.B. and Arikan, O., "Regularized estimation of VTEC from GPS data for a desired time period", Radio Science, vol. 39(6), RS6012, 2004.

[3] Nayir, H., Arikan, F., Arikan, O. and Erol, C.B., "Total electron content estimation with Reg-Est", Journal of Geophysical Research-Space Physics, vol. 122, A11313, 2007.

[4] Arikan, F., Nayir, H., Sezen, U., Arikan, O., "Estimation of single station interfrequency receiver bias using GPS-TEC", Radio Science., vol.43, RS4004, 1-13, 2008.

[5] N.Turel, and F. Arikan, "Probability density function estimation for characterizing hourly variability of ionospheric total electron," Radio Science, vol. 45, RS6016, 1-10, 2010.

[6] T.L. Gulyaeva, "Storm time behavior of topside scale height inferred from the ionosphere-plasmasphere model driven by the F2 layer peak and GPS-TEC observations," Advances in Space Research, doi: 10.1016/j.asr.2010.10.025, 2010 